\title{
Classification of Liver Cirrhosis on m-Mode Ultrasound Images by Extended Higher Order Local Autocorrelation Features
}

\author{
Yoshihiro Mitani, Yusuke Fujita, Yoshihiko Hamamoto, and Isao Sakaida
}

\begin{abstract}
Ultrasound images are widely used for diagnosis of liver cirrhosis. In liver cirrhosis classification on M-mode ultrasound images, the use of higher order local auto-correlation (HLAC) features has been shown to be effective. In the previous study, we used the traditional 25 dimensional HLAC features. The 25 HLAC features are made by 25 mask patterns with up to 0th, 1st, and 2nd-order. On the other hand, there exists an extension of HLAC features. The extended HLAC features were shown to be more effective when higher-order HLAC features were used. Therefore, by the use of the extended HLAC features, we expected the liver cirrhosis classification performance to improve. However, the effectiveness of the extended HLAC features to classify the liver cirrhosis images is not clear. In this paper, more effectively to classify liver cirrhosis M-mode ultrasound images, we examine the performance of extended HLAC features.
\end{abstract}

Index Terms-Liver cirrhosis classification, M-mode ultrasound images, HLAC features, extended HLAC features.

\section{INTRODUCTION}

Ultrasound images are widely used for diagnosis of liver cirrhosis. The liver cirrhosis classification method on M-mode ultrasound images is required. Fig. 1 shows M-mode ultrasound images. Fig. 1(a) is normal. On the other hand, (b) is cirrhosis. In the previous study, Zhou's method has been proposed [1]. This method consists of 2 processes. Firstly, an abdominal aorta wall from the M-mode ultrasound image is extracted. Secondly, the feature vector generated by based on the extracted abdominal aorta wall is used for liver cirrhosis classification. Hayashi et al. have also proposed a method to extract an abdominal aorta wall accurately [2]. The method of Hayashi et al. is based on a weighted coefficient of correlation of the abdominal aorta wall. It outperforms the Zhou's method. However, Zhou's and Hayashi et al. approaches depend on the accuracy of the abdominal aorta wall extraction If the extraction of the abdominal aorta wall fails, the subsequent liver cirrhosis classification fails by all means. Therefore, we have examined a method to classify the liver cirrhosis not using the abdominal aorta wall extraction process [3], [4]. In the proposed method, we used higher-order local auto-correlation (HLAC) [5] features. The HLAC features are successfully applied to pattern recognition

Manuscript received October 9, 2014; revised February 10, 2015.

Yoshihiro Mitani is with the Department of Intelligent System Engineering, National Institute of Technology, Ube College, Ube, Japan (e-mail: mitani@ube-k.ac.jp).

Yusuke Fujita, Yoshihiko Hamamoto, and Isao Sakaida are with Yamaguchi University, Ube, Japan. problems. The advantages of the HLAC features are considered to be simple, robust, and easily implemented. Furthermore, in order to improve the liver cirrhosis classification performance, we have also proposed to apply image processing techniques [6] of a thresholding technique and a shading method [3], [4]. These methods are expected effectively to reduce noises in the image. In general, the ultrasound images have heavy noises. By the combination of the adaptive thresholding and a shading technique, the HLAC feature vector was effectively extracted [3], [4].

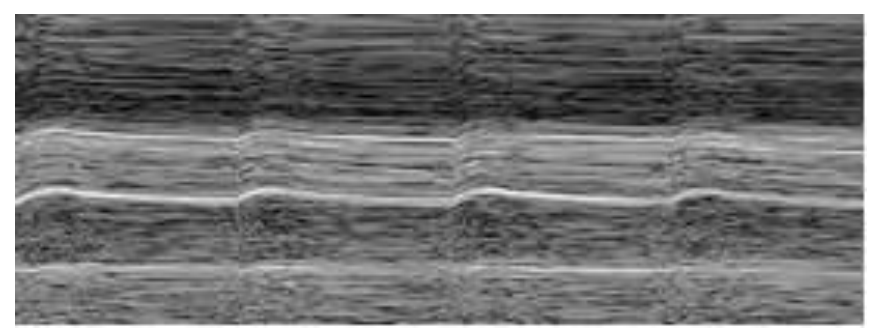

(a) Normal

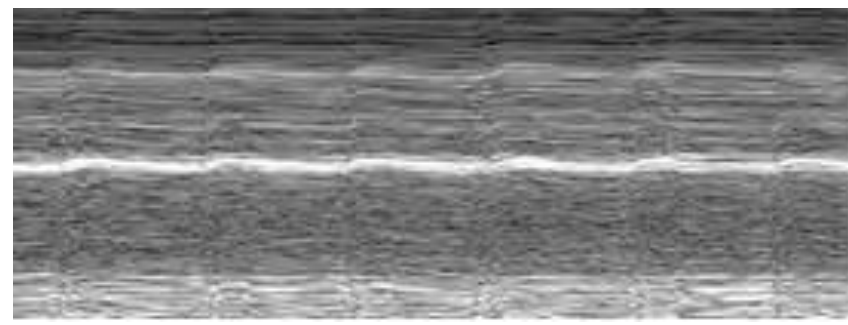

(b) Cirrhosis

Fig. 1. M-mode ultrasound images.

The dimensionality of the traditional HLAC feature vector is 25 . The 25 HLAC feature vector is produced by 25 mask patterns with up to 0th, 1st, and 2nd-order. In the paper [7], the extended HLAC feature vector approach was proposed. The HLAC features are extended by from zeroth-order to eighth-order. This means the dimensionalities of the HLAC features are from 1 to 223 . The experimental results showed the effectiveness of the higher-order HLAC features for texture image classification [7]. The higher dimensionality is, the more classification performance improves. That is, it is expected that the more complicated the mask patterns are, the richer the classification information becomes. Therefore, we expected the extended HLAC feature vector approach to further improve the liver cirrhosis classification performance on the M-mode ultrasound images. In this paper, we examine the extended HLAC feature vector approach. Experimental result shows a surprised result. The lower-order HLAC feature shows effective. Thus, in the liver cirrhosis M-mode ultrasound image classification by the use of the HLAC 
feature vector approach, the more primitive feature should be used.

\section{A Previous WORK}

In the previous study [3], [4], we have proposed a method of liver cirrhosis classification on M-mode ultrasound images by HLAC features [5]. The traditional HLAC features are obtained by the use of $253 \times 3$ mask patterns. The dimensionality of the HLAC feature vector is 25 . The HLAC feature-based method is expected to be effective for shapes, such as the abdominal aorta wall, to get information of a local direction element.

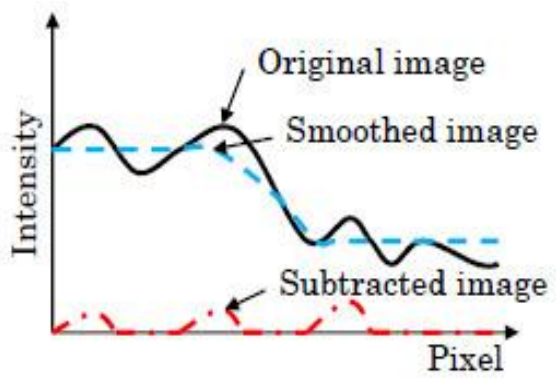

Fig. 2. A shading technique.

Furthermore, in order to effectively to use HLAC features, we have also proposed to apply image processing techniques [6]. The effective image processing techniques were a shading technique and the adaptive thresholding [3], [4]. Fig. 2 shows a shading technique. Fig. 3 shows images with a shading technique and an adaptive thresholding technique.

A shading technique reduces heavy noises in the ultrasound image. Fig. 2 illustrates a shading technique. First, the smoothed image (Fig. 3(b)) is generated by applying a median filter to the original image (Fig. 3(a)). Second, the subtracted image (Fig. 3(c)) is produced by subtracting the smoothed image to the original image. If a value of the subtracted image is less than zero, we regard the value as zero. This means that these values are ignored. Here, we show a numerical expression. Assume that the original image, smoothed image, and subtracted image are $f_{i j}, g_{i j}$, and $h_{i j}$, respectively. The subtracted image with a shading technique is generated as follows:

$$
h_{i j}=f_{i j}-g_{i j} \text {, if } h_{i j}<0 \text { then } h_{i j}=0 .
$$

Finally, we carry out the adaptive thresholding to the subtracted image. Here, the selection of the median filter size is important. Since, it influences the smoothing degree. The median filter size should be carefully selected.

By the adaptive thresholding, the HLAC features more effectively extract the classification information. The adaptive thresholding technique finds local threshold in each pixel. In the adaptive thresholding technique, we use the mean of the local intensity distribution. If the pixel is larger than the mean, we regard the pixel as the white pixel. Otherwise, the pixel is black. The size of local areas in the adaptive thresholding directly influences the liver cirrhosis classification performance. We have to determine the size of local areas in the adaptive thresholding to separate desirable foreground objects from the background. Fig. 3(d) shows a binary image by an adaptive thresholding technique. In Fig. $3(\mathrm{~d})$, the size of the local areas in the adaptive thresholding is $13 \times 13$.

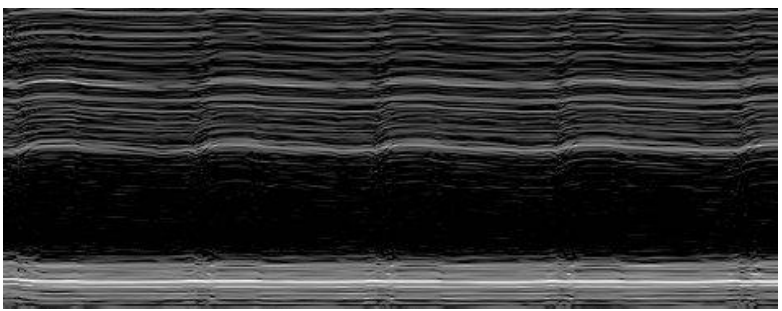

(a) Original gray image

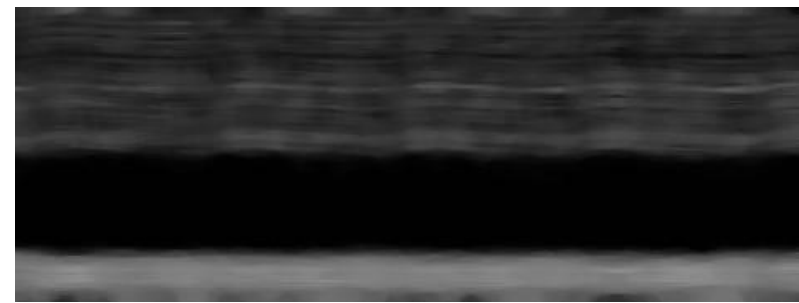

(b) Smoothed image

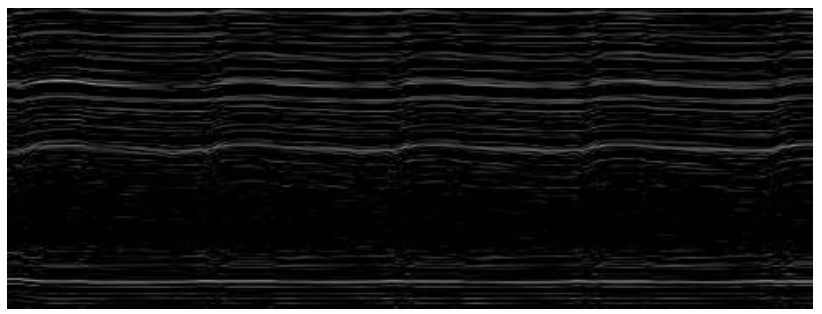

(c) Subtracted image

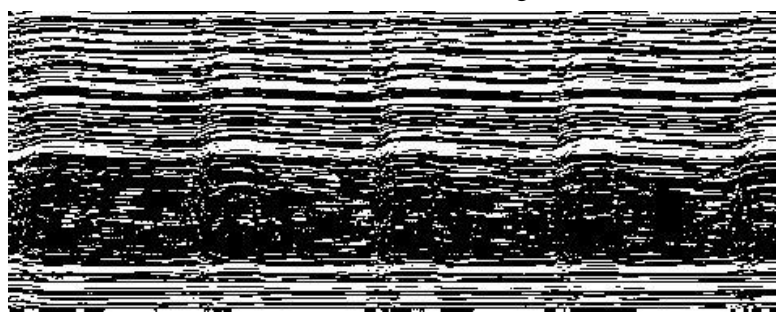

(d) Binary image by an adaptive thresholding technique Fig. 3. Images with a shading technique and an adaptive thresholding technique.

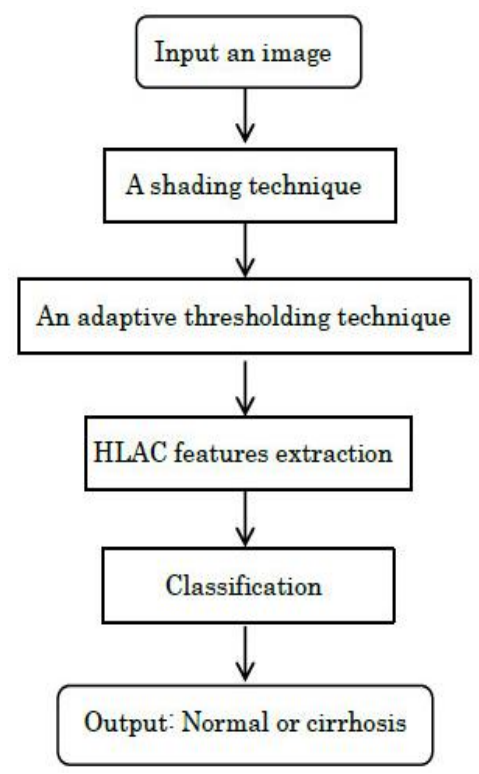

Fig. 4. A proposed method.

Note that the sizes of the median filter and local areas in the 
adaptive thresholoding influence the liver cirrhosis classification performance. The experimental result gives a favorable result [4]. The error rate is $17.1 \%$. Both sizes of the median filter and local areas in the adaptive thresholding are the same, $15 \times 15$, shows a minimum. Then the dimensionality of the traditional HLAC features is 25 .

\section{A PROPOSED METHOD}

Fig. 4 shows a proposed method. First, an ultrasound image turns into a binary image carried out by a shading technique and an adaptive threshold. Second, the HLAC features are extracted from this binary image. Finally, by a classifier, we obtain an output result: normal or cirrhosis. In the previous study [3], [4], we showed the effectiveness of a shading technique and an adaptive thresholding technique. In this paper, we focus the HLAC features extraction process.

In order to further improve the classification performance of the liver cirrhosis on M-mode ultrasound images, we explore the extended HLAC features [7]. The traditional HLAC feature vector is 25 dimensions. The 25 mask patterns consist of zeroth-order, first-order, and second-order HLAC features with 3 times 3 pixels. In this paper, we use the extended HLAC features with up to third-order. Fig. 5 shows 70 mask patterns of the zeroth-order to third-order HLAC features $(3 \times 3$ pixels $)$. The figure is cited from the literature [7] and we write down 70 mask patterns with up to third-order HLAC features. In the experiment, we examine the classification performance of up to first-order, second-order, and third-order HLAC features. The dimensionality is from 5 to 70. In Fig. 5, 5, 25, and 70 mask patterns with up to first-, second-, and third- order HLAC features are 5, 25, and 70 dimensions, respectively. The HLAC features are obtained by the following: First, each of red-colored pixels corresponding to the original image is counted, and accumulated in overall the image. Note that if the pixels do not correspond to the red-colored pixels, we don't count. Second, the accumulated value is divided by the image size. This means normalization. Then the value is obtained as a feature of a HLAC mask. If the value is higher, the more strongly the mask shape appears. On the other hand, the less the value is, the more weakly the mask pattern appears.
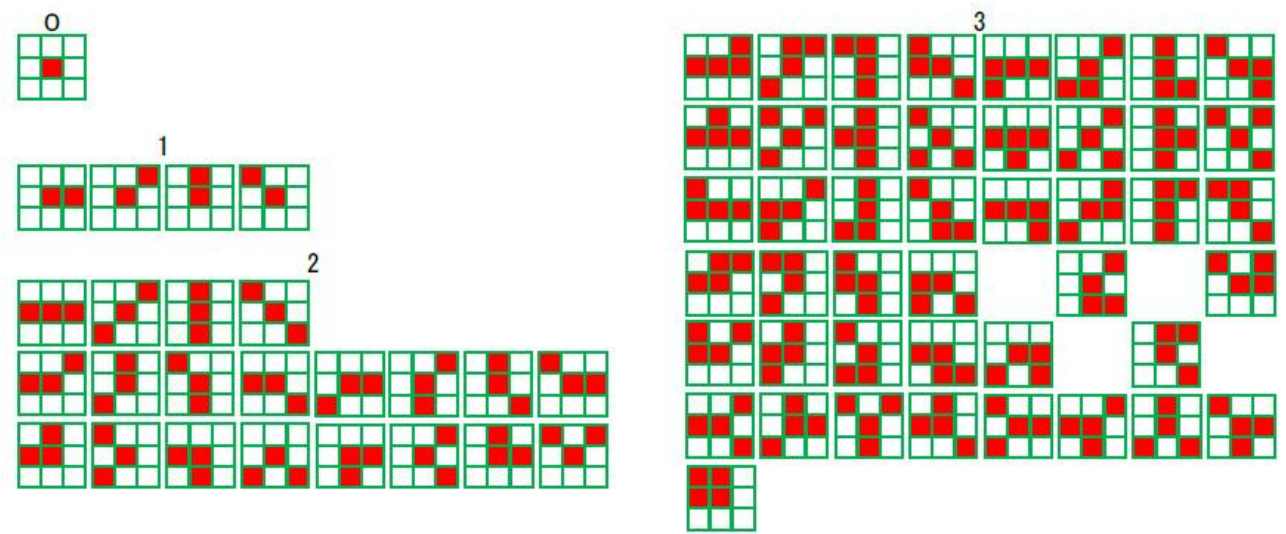

Fig. 5.70 mask patterns of the zeroth-order to third-order HLAC features $(3 \times 3$ pixels).

\section{EXPERIMENTAL RESULTS}

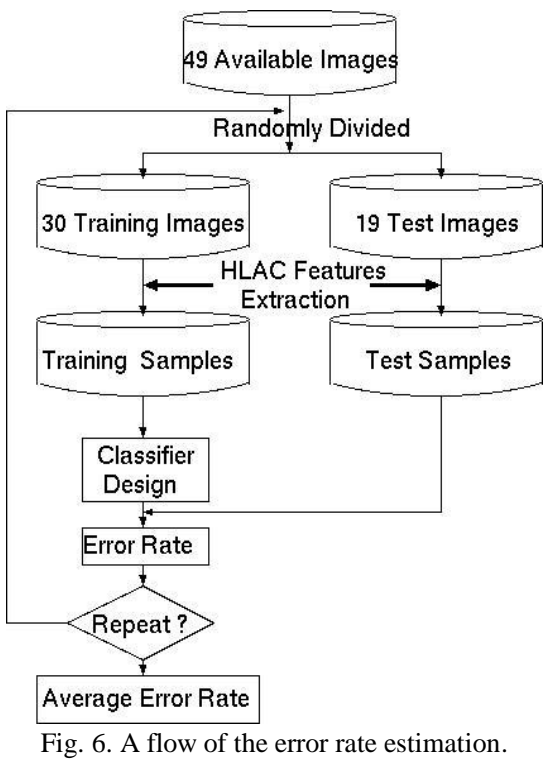

In the experiments [3], [4], we used 49 available M-mode ultrasound images: 28 cirrhosis images and 21 normal images. This is a two-class problem. The gray level is 8 bits, i.e., 256. In the size of images, height times width equals to about 180 pixels times 250-570 pixels. The effectiveness of the proposed method is examined in terms of the error rate. The error rate $P_{e}$ is defined as follows:

\section{$P_{e}=100 \times$ No. of test samples misclassified / No. of all test samples [\%]}

In error rate estimation literature, the holdout method has been successfully used, because it maintains the statistical independence between the training and test samples [8]. In order to evaluate the proposed method, the average error rate was obtained by the holdout method. Fig. 6 shows a flow of the error rate estimation. First, we randomly divided 49 available images into 30 training images and 19 test images. 30 training images consist of 15 cirrhosis and 15 normal images. On the other hand, 19 test images are made up of 13 cirrhosis and 6 normal images. Second, we extracted HLAC features from these images. Third, the error rate was computed by using nearest neighbor classifier [8]. Finally, by 100 repetitions, the average error rate was obtained. 
TABLE I: AVERAGE ERROR RATE OF UP TO FIRST-ORDER, SECOND-ORDER, AND THIRD-ORDER HLAC FEATURES

\begin{tabular}{|l|l|l|}
\hline Order & Dimensionality & Average error rate $\%)$ \\
\hline 0,1 & 5 & 12.4 \\
\hline $0,1,2$ & 25 & 17.1 \\
\hline $0,1,2,3$ & 70 & 23.6 \\
\hline
\end{tabular}

The purpose of the experiment 1 is to investigate the performance of the extended HLAC features. We vary the dimensionality. Fig. 7 shows an outline of the experiment 1 . Table $I$ is the result of the experiment 1 on the minimum average error rate. The minimum average error rate means the optimal sizes of local areas in the adaptive thresholding and the median filter in terms of the average error rate. From the results, contrary to our expectations, the average error rate of lower-order HLAC features shows a minimum. The 5-dimensional HLAC feature vector is the better. The optimal sizes of local areas in the adaptive thresholding and the median filter are $15 \times 15$ and $17 \times 17$, respectively. In the liver cirrhosis classification by the HLAC feature vector based method, more primitive HLAC feature works better. The following experiment is carried out by 5-dimensional HLAC

\section{feature vector.}

The purpose of the experiment 2 is to investigate the influence of sizes of local areas in the adaptive thresholding and the median filter. Fig. 8 shows an outline of the experiment 2. Table II shows the influence of sizes of local areas in the adaptive thresholding and the median filter on the average error rate. These sizes must be optimized in terms of the error rate.

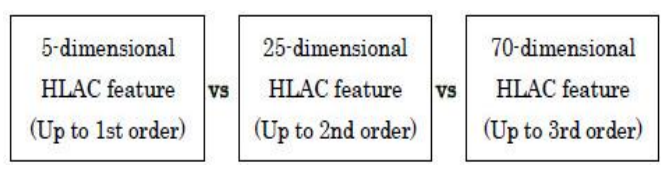

Fig. 7. An outline of the experiment 1.

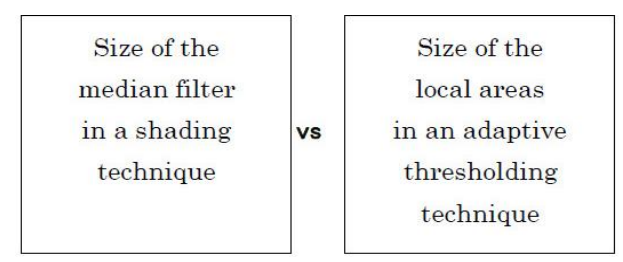

Fig. 8. An outline of the experiment 2.

TABLE II: INFLUENCE OF SizeS OF THE MEDIAN FiLTER AND LOCAL AREAS IN THE ADAPTIVE THRESHOLDING ON THE AVERAGE ERROR RATE (\%)

\begin{tabular}{|c|c|c|c|c|c|c|c|}
\hline \multirow{2}{*}{$\begin{array}{l}\text { Size of local areas } \\
\text { in the adaptive thresholding }\end{array}$} & \multicolumn{7}{|c|}{ Size of the median filter } \\
\hline & $7 \times 7$ & $9 \times 9$ & $11 \times 11$ & $13 \times 13$ & $15 \times 15$ & $17 \times 17$ & $19 \times 19$ \\
\hline $7 \times 7$ & 16.3 & 17.0 & 16.6 & 16.7 & 17.0 & 15.0 & 18.2 \\
\hline $9 \times 9$ & 13.3 & 13.4 & 14.7 & 13.1 & 13.1 & 13.0 & 14.1 \\
\hline $11 \times 11$ & 15.5 & 15.9 & 18.2 & 15.8 & 15.7 & 16.0 & 16.3 \\
\hline $13 \times 13$ & 15.9 & 16.1 & 16.6 & 14.7 & 14.7 & 14.3 & 15.2 \\
\hline $15 \times 15$ & 14.0 & 14.0 & 14.6 & 12.6 & 12.6 & 12.4 & 13.2 \\
\hline $17 \times 17$ & 16.7 & 15.8 & 16.1 & 13.5 & 13.6 & 13.6 & 13.8 \\
\hline
\end{tabular}

\section{CONCLUSIONS}

In this paper, more effectively to classify liver cirrhosis M-mode ultrasound images, we examined the performance of extended HLAC features. The extended HLAC features are expected to improve the classification performance of the liver cirrhosis images. Contrary to our expectations, the experimental results show the effectiveness of the lower dimensional HLAC features. The classification performance of only 5 dimensional HLAC features outperforms those of both 25 and 70 dimensional HLAC features. Thus, in the liver cirrhosis classification by the HLAC feature vector based method, more primitive HLAC feature works better. In the future, we investigate the lower dimensional HLAC features in detail.

\section{ACKNOWLEDGMENT}

This work was supported by JSPS KAKENHI Grant Number 25330357.

\section{REFERENCES}

[1] G. Zhou, Y. Wang, W. Wang, Y. Sun, and Y. Chen, "Decision of cirrhosis using liver's ultrasonic images," in Proc. the 2005 IEEE 27th Annual Conference on Engineering in Medicine and Biology, Shanghai, 2005, pp. 3351-3354.

[2] T. Hayashi, Y. Fujita, Y. Mitani, Y. Hamamoto, M. Segawa, S. Terai, and I. Sakaida, "An abdominal aorta wall extraction for liver cirrhosis classification using ultrasonic images," in Proc. 2011 International
Symposium on Computational Models for Life Sciences, Toyama, 2011, pp. 343-344.

[3] K. Fujino, Y. Mitani, H. Hayashi, Y. Fujita, Y. Hamamoto, M. Segawa, S. Terai, and I. Sakaida "A note of liver cirrhosis classification on M-mode ultrasound images by higher-order local auto-correlation features," in Proc. International Conference on Soft Computing and Pattern Recognition, Hanoi, 2013, pp. 50-53.

[4] K. Fujino, Y. Mitani, Y. Fujita, Y. Hamamoto, and I. Sakaida, "Liver cirrhosis classification on M-mode ultrasound images by higher-order local auto-correlation features," Journal of Medical and Bioengineering, vol. 3, no. 1, pp. 29-32, 2014.

[5] N. Otsu, and T. Kurita, "A new scheme for practical flexible and intelligent vision systems," in Proc. IAPR Workshop on Computer Vision, Special Hardware and Industrial Applications, 1988, pp. 431-435.

[6] J. C. Russ, The Image Processing Handbook, Third Edition, CRC Press, 1999.

[7] T. Toyoda and O. Hasegawa, "Extension of higher order local autocorrelation features," Pattern Recognition, vol. 40, pp. 1466-1473, 2007.

[8] K. Fukunaga, Introduction to Statistical Pattern Recognition, Second Edition, Academic Press, 1990.

Yoshihiro Mitani is currently a professor at National Institute of Technology, Ube College, Japan. His research interests include pattern recognition and image processing techniques. He is a member of IEEE.

Yusuke Fujita is currently an associate professor at Yamaguchi University, Japan. His research interests include pattern recognition and image processing techniques.

Yoshihiko Hamamoto is currently a professor at Yamaguchi University, Japan. His research interest is pattern recognition. He is a member of IEEE. 